\title{
Enhanced Mid -Infrared Reflectance with Graphene Coated Silicon Carbide Nanowires
}

\author{
Patrick Rufangura ${ }^{1}$, Arti Agrawal ${ }^{1,2}$, Matteo Bosi ${ }^{3}$, Thomas G. Folland ${ }^{4}$, \\ Joshua D. Caldwell ${ }^{4}$ and Francesca Iacopi ${ }^{1},^{2}$ \\ 1 School of Electrical and Data Engineering, Faculty of Engineering and IT, \\ University of Technology Sydney, Broadway, 2007 NSW, Australia \\ 2 Australian Research Council Centre of Excellence on Transformative Meta-Optical Systems, \\ University of Technology Sydney, Broadway, 2007 NSW, Australia \\ 3 IMEM-CNR Institute, Parco Area delle Scienze 37/A, 43124 Parma, Italy \\ 4 Department of Mechanical Engineering, Vanderbilt University, Nashville, TN 37212, USA
}

\begin{abstract}
The mid-infrared (MIR) optical spectrum hosts a variety of sought- after photonic applications. Herein we simulate and experimentally demonstrate reflectance enhancement of MIR light using graphene-coated silicon carbide nanowires on silicon, showing promise for on-chip MIR nanophotonics. (C) 2020 The Author(s)
\end{abstract}

The unique optical properties of graphene, which stem from its 2D nature, make it a highly promising material for applications in integrated, miniaturized photonics [1, 2]. Although the transfer of graphene on diverse substrates and pre-fabricated devices is possible [3,4], a more direct alternative capable of being scaled -up to large surfaces, is the growth of epitaxial graphene on a semiconductor like silicon carbide (EG/SiC) [5-8]. Graphene and $\mathrm{SiC}$ have been extensively, but separately, studied for MIR and terahertz (THz) photonics, respectively $[9,10]$. The $\mathrm{EG} / \mathrm{SiC}$ is an ideal platform for investigating exciting physics such as the coupling between graphene plasmons and optic phonons in $\mathrm{SiC}$, which could lead to free-space IR light capture and onchip manipulation with low-losses and superior confinement $[11,12]$. However, the amount of experimental data is still limited in this field.

In this work, we use attenuated total reflectance Fourier transformed infrared (ATR-FTIR) spectroscopy, in combination with electromagnetic simulations using the finite element method (FEM), to reveal a pronounced reflectance enhancement in the MIR when graphene is coated on SiC nanowires (NWs; Fig. 1). The SiC NWs are grown on a silicon substrate as a nanowire forest (see inset in Fig.1a), with an average diameter around 30$50 \mathrm{~nm}$, and are subsequently graphitized according to our catalytic alloy method [13]. In the FEM, a single nanowire of $50 \mathrm{~nm}$ diameter was simulated with periodic boundary conditions. The graphene was simulated as an infinitesimal thin conductive layer $(0.33 \mathrm{~nm})$ using a transition boundary condition. A TM/p- polarized electromagnetic source was used in the simulation (see Fig. 1c).

From both experimental and simulated spectra, a very low reflectance is found for bare SiC NWs (Fig. 1 blue line), confirmed by the simulated weak electric field intensities (Fig. 2 a-c). This is expected due to the small size and limited amount of SiC NWs being unable to absorb enough light. However, a sharp enhancement in reflectance for the SiC NWs is observed, once they are graphitized (Fig.1a, red line).

The simulation performed on graphene-coated $\mathrm{SiC}$ NW/Si (Fig.1b black line) reveals two modes with a substantial field enhancement as compared to bare SiC NWs (Fig. 2 d-f). Our previous observation of this material system indicated the presence of a low-density oxide layer between graphene and SiC NWs [13]. After including an oxide layer in our graphene/SiC NW/Si FEM model, we observe an additional mode and further reflectance enhancements (Fig 1b, red line). There is a satisfactory similarity with the spectra measured experimentally. The field profiles of the electric fields for the different modes (Fig $2 \mathrm{~g}-\mathrm{i}$ ) show maximum field 
intensities occurring at the interfaces (oxide/SiC NW and graphene/oxide). This extraordinary surface enhancement of about one order of magnitude may be originating from the coupling between graphene plasmon and $\mathrm{SiC}$ phonon polariton. We also suggest that the oxide layer could potentially act as a coupling medium.

(a)

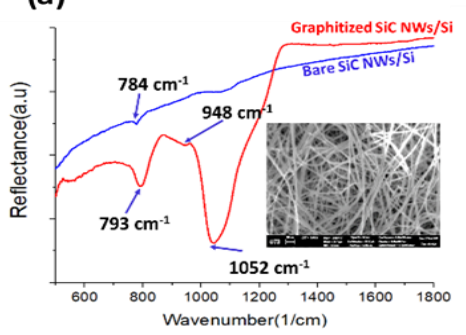

(b)

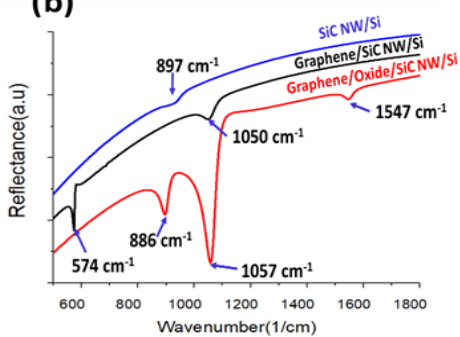

(c)

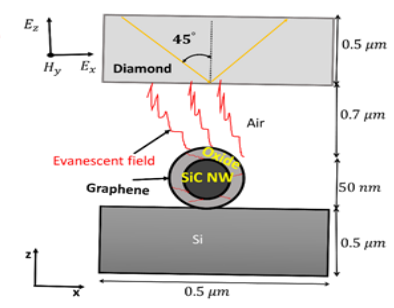

Figure 1. (a) Measured reflectance on bare SiC NWs/Si (blue line), and graphitized SiC NWs/Si (red line), inset shows the SEM image of $\mathrm{SiC}$ nanowires/Si. (b) Simulated reflectance on bare $\mathrm{SiC} \mathrm{NW} / \mathrm{Si}$ (blue line), graphene/SiC NW/Si (black line), and graphene/oxide/SiC $\mathrm{NW} / \mathrm{Si}$ (red line). (c) The schematic of the simulation model with one SiC NW coated with oxide and graphene.

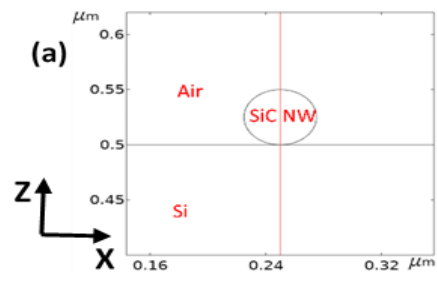

(b) $\times 10^{5}$

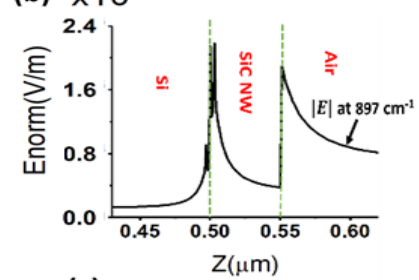

(c)

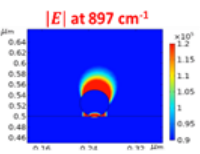

(d)

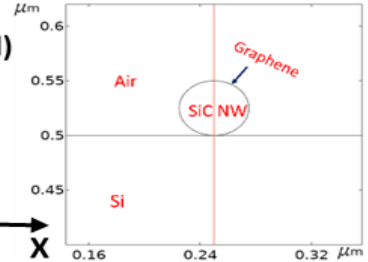

(e)

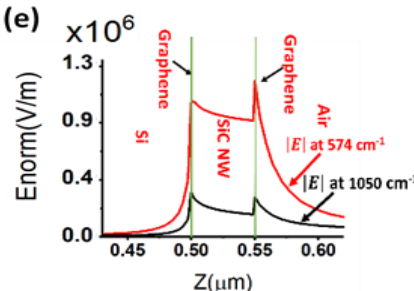

(f)

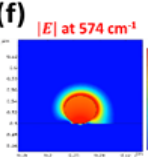

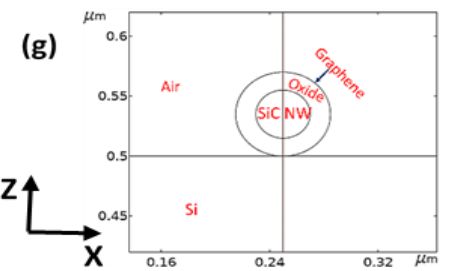

(h)
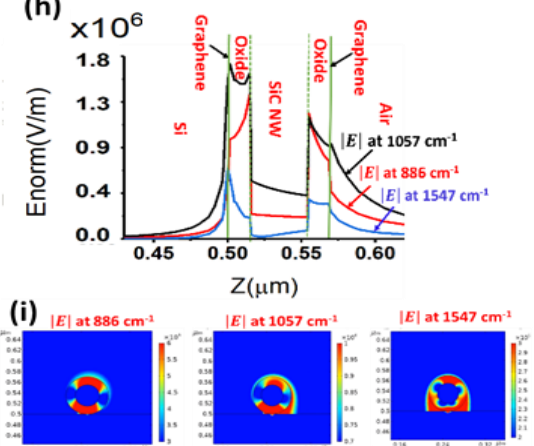

Figure 2. Calculated electric fields along a cut line (Z) and the field map for electromagnetic modes in (a-c) $\mathrm{SiC} N W s / S i$, (d-f) graphene/SiC NW/Si, and (g-i) graphene/oxide/SiC NW/Si.

\section{References}

[1] A. K. Geim, "Graphene: status and prospects," science, vol. 324, no. 5934, pp. 1530-1534, 2009.

[2] K. S. Novoselov et al., "Electric field effect in atomically thin carbon films," science, vol. 306, no. 5696, pp. 666-669, 2004.

[3] L. Xiong et al., "Photonic crystal for graphene plasmons," Nature communications, vol. 10, no. 1, pp. 1-6, 2019.

[4] J. D. Caldwell et al., "Technique for the dry transfer of epitaxial graphene onto arbitrary substrates," ACS nano, vol. 4, no. 2, pp. 1108-1114, 2010.

[5] B. V. Cunning, M. Ahmed, N. Mishra, A. R. Kermany, B. Wood, and F. Iacopi, "Graphitized silicon carbide microbeams: waferlevel, self-aligned graphene on silicon wafers," Nanotechnology, vol. 25, no. 32, p. 325301, 2014.

[6] W. A. De Heer et al., "Large area and structured epitaxial graphene produced by confinement controlled sublimation of silicon carbide," Proceedings of the National Academy of Sciences, vol. 108, no. 41, pp. 16900-16905, 2011.

[7] W. A. De Heer et al., "Epitaxial graphene," Solid State Communications, vol. 143, no. 1-2, pp. 92-100, 2007.

[8] A. Pradeepkumar et al., "p-type Epitaxial Graphene on Cubic Silicon Carbide on Silicon for Integrated Silicon Technologies," ACS Applied Nano Materials, 2019.

[9] X. Luo, T. Qiu, W. Lu, and Z. Ni, "Plasmons in graphene: recent progress and applications," Materials Science and Engineering: R: Reports, vol. 74, no. 11, pp. 351-376, 2013.

[10] J. D. Caldwell et al., "Low-loss, infrared and terahertz nanophotonics using surface phonon polaritons," Nanophotonics, vol. 4 , no. 1, pp. 44-68, 2015.

[11] R. Koch et al., "Robust phonon-plasmon coupling in quasifreestanding graphene on silicon carbide," Physical review letters, vol. 116, no. 10, p. 106802, 2016.

[12] Y. Liu and R. F. Willis, "Plasmon-phonon strongly coupled mode in epitaxial graphene," Physical Review B, vol. 81, no. 8, p. 081406, 2010.

[13] N. Mishra, M. Bosi, F. Rossi, G. Salviati, J. Boeckl, and F. Iacopi, "Growth of graphitic carbon layers around silicon carbide nanowires," Journal of Applied Physics, vol. 126, no. 6, p. 065304, 2019. 